\title{
Small Diameter Coronary Arteria Designed In Vitro
}

\section{Brett Isenberg C}

Departments of Chemical Engineering \& Materials Science, University of Minnesota, Minneapolis, MN 55455, USA

*Corresponding author: Brett Isenberg C, Departments of Chemical Engineering \& Materials Science, University of Minnesota, Minneapolis, MN 55455, USA, Tel: 612-625-6868; E-mail:tranquillo@cems.umn.edu

Rec date: Sep 16, 2014; Acc date: Dec 20, 2014; Pub date: Dec 23, 2014

Copyright: (C) 2014 Brett IC. This is an open-access article distributed under the terms of the Creative Commons Attribution License, which permits unrestricted use, distribution, and reproduction in any medium, provided the original author and source are credited.

\begin{abstract}
Although the necessity for a useful blood vessel replacement is evident, the lower blood flow velocities of smalldiameter arteries just like the arterial blood vessel have semiconductor diode to the failure of artificial materials that are triple-crown for large-diameter grafts. Though autologous vessels stay the quality for little diameter grafts, several patients don't have a vessel appropriate to be used owing to vascular unwellness, amputation, or previous harvest. As a result, tissue engineering has emerged as a promising approach to deal with the shortcomings of current therapies. Investigators have explored the utilization of blood vessel tissue cells or differentiated stem cells combined with numerous kinds of natural and artificial scaffolds to form cannular constructs and subject them to chemical and/or mechanical stimulation in an endeavor to develop a useful small-diameter blood vessel replacement graft with variable degrees of success. Here, we tend to review the progress all told these major sides of the sector.
\end{abstract}

\section{Introduction}

In 2002, over five hundred 000 surgical procedures were performed involving replacement of small-caliber blood vessels. Despite a transparent clinical want for a useful blood vessel graft, success has been restricted to blood vessel replacements of large-caliber vessels like the body part and aorta, arch vessels, iliac, and customary leg bone arteries; but, small-caliber $(<6 \mathrm{~mm})$ blood vessel substitutes, that account for a majority of the demand, have usually tried inadequate mostly thanks to acute thrombogenicity of the graft, conjugation tissue layer dysplasia, aneurism formation, infection, and progression of arteriosclerosis\} disease. The lower blood flow velocities of smaller vessels cause a unique set of standard and introduce a number of latest issues not encountered in large-caliber blood vessel substitutes wherever polyester and distended polytetrafluorethylene grafts have succeeded. Though autologous vessels, like the vena, stay the quality for tiny diameter grafts, several patients don't have a vessel appropriate to be used thanks to tube-shaped structure malady, amputation, or previous harvest [1-3].

\section{Fabrication Methods}

Decellularized tissues have the advantage of being entirely composed of natural living thing matrix (ECM), giving them various blessings in mechanical properties and biocompatibility. 15 not like the opposite main approaches, these grafts are generally planted while not cells gift with the idea that they're going to be recellularized by host cells following implantation. These tissues are often either vascular or nonvascular (eg, little enteric connective tissue [SIS]) in origin. Decellularization is usually accomplished by treating tissues with a mix of detergents, catalyst inhibitors, and buffers. The plant tissue advantages from grossly retentive the structure and composition of a native vessel following decellularization; but, decellularization will adversely impact the tissue, leading to reduced final durability and compliance[4].Vital shrinkage is usually determined in decellularized vessels, presumptively as a result of proteoglycans being far away from the tissues by the detergent treatment.20 Decellularized xenografts bear aneurism formation, infection, and occlusion. Additionally, their residual antigenicity will impair succeeding re-endothelialization.

\section{Biodegradable Polymer Scaffolds}

Several perishable artificial chemical compound scaffolds are investigated for his or her quality in plant tissue engineering applications. The essential plan altogether of those approaches is to seed cells onto a degradable chemical compound scaffold that supports tissue growth and transforming. As a result of the conditions accustomed produce them are too harsh for the cells to survive, cells cannot be directly entrapped throughout scaffold formation. Cellularization is later on accomplished by looking forward to cellinvasion or cell-seeding techniques that will cause suboptimal cell distribution. Ideally, the chemical compound are slowly resorbed in culture or when implantation, departure solely the tissue generated by the cells [5]. These polymers are advantageous as a result of their microstructure, mechanical properties, and organic process rates are often fastidiously controlled via chemical composition in a shot to reinforce tissue growth and transforming. moreover, these scaffolds will give initial mechanical perform for the graft in vitro and/or in vivo till the cells have the chance to synthesize vital amounts of ECM; but, premature implantation could be a major risk as a result of a failure of the cells to provide the requisite electronic warfare before chemical compound degradation would be harmful.

\section{Cell Sheets}

Perhaps the foremost spectacular utterly biological tissueengineered graft style so far, a minimum of in terms of burst pressure achieved with human cells, is that the cell sheet approach developed by Auger and colleagues. During this approach, sheets of low passage human infant SMCs were mature on culture plates within the presence of elevated antioxidant (to induce important albuminoid synthesis). Once spare time a sheet was far from its culture plate and wrapped around a porous, hollow spindle to make the media of the tissue construct (the porous spindle allowed transport of nutrients to the inner surface of the membrane by flowing substance through the 
spindle lumen) [6-8]. Within the same manner, a sheet of fibroblasts was mature and wrapped round the media to supply the tunic. Once many weeks of maturation, these two layers amalgamate into one cohesive layer. At now, the hollow constructs were far from the mandrels and seeded with ECs on the Luminal surface by cannulating the ends, filling the lumen with an answer of ECs, and so slowly rotating them long.

However, though these tissue-engineered blood vessels area unit a lot of compliant than expanded polytetrafluorethylene grafts, they're apparently abundant less compliant than the small-caliber vessels they're designed to exchange, that may doubtless cause complications associated with the difficulty of compliance couple, which frequently ends up in conjugation tissue layer dysplasia. This lack of compliance could also be thanks to shy elastic fiber deposition, which can conjointly cause unretrievable creep predisposing cardiovascular disease formation. In spite of this, short-run affixation experiments in a very canine model were very encouraging. In a very ensuant study, vessels created during this manner were shown to own marked vasoactive responses to various agonists like aminoalkane, bradykinin, ATP and UTP.

\section{Biopolymer Scaffolds}

Motivated by the pioneering work of Weinberg and Bell, variety of researchers have investigated the prospect of seeding ECs onto the phenobarbitone surface of a tube of reconstituted kind I albuminoid gel inhabited and compacted by SMCs to construct a totally biological tube graft. The thought of constructing such a graft, termed a "bioartificial artery" (BAA), during this manner is extraordinarily appealing. Within the case of albuminoid, it's the foremost copious super molecule within the organic structure and is that the major electronic countermeasures part in most tissues, together with the blood vessel wall, creating it a natural cell substrate [9]. Bovine kind I albuminoid is already Associate in Nursing approved material for implantation and utilized in the America Food and Drug Administration-approved bioartificial skin product Apligraf (Organogenesis Inc). Fibrin, another unremarkably used biopolymer, is that the major structural super molecule in blood clots and plays a significant role within the sequent wound healing response, being transforming into cell-derived electronic countermeasures.

\section{Novel Biomimetic Materials}

A new generation of biomaterials is being developed that tries to mimic the structure and characteristics of native electronic countermeasures, like fibrillar structure, viscoelasticity, cell adhesion domains, protein binding, and chemical change sensitivity. Such materials ar engaging as a result of, in essence, their properties are often promptly controlled whereas mimicking several of the vital biological functions of the native electronic countermeasures, that ar mostly lacking from artificial polymers like PGA. The technique of electrospinning has been accustomed manufacture fibers with diameters on the order of these found in native electronic countermeasures from polyester in addition as biopolymers like albuminoid and factor I. though directly yielding circumferential alignment (or a prescribed alignment pattern), cells can't be entrapped throughout electrospinning and should be seeded within the scaffolds postfabrication [10-13]. In an effort to develop materials for facilitating blood vessel healing following balloon surgery, Seliktar et al. were able to enhance Common Market migration and adhesion on polythene glycol hydrogels that incorporated cell-adhesion domains and domains that were sensitive to matrix metalloproteinase (MMP)-2 cleavage additionally to tethering vascular epithelium protein, that promotes MMP-2 expression in ECs, to the chemical compound backbone. an analogous gel has been developed that comes with celladhesion and plasmin-degradable domains supported clotting factor and anti-thrombin III, yet as Lipo-Hepin-binding sites to encourage the deposition of heparin and, in turn, heparin-binding growth factors. Though of these techniques area unit promising, these materials have however to be place into apply as potential scaffolds for plant tissue engineering [14].

\section{Mechanical Conditioning via Bioreactors}

Despite several advances in plant tissue engineering, investigators have however to develop a useful blood vessel replacement graft that not solely meets the wants for mechanical strength and stiffness however additionally mimics the biological practicality of a native vessel. various approaches are taken to handle these problems as well as, within the case of biopolymer-based fabrication, catalyst crosslinking (glycation) victimisation carbohydrate, catalyst cross-linking victimization lysyl enzyme, and fabricating fibrin-based MEs with TGF- $\beta 1$ and hormone matter supplementation as another means that of stimulating ECM synthesis and cross-linking[15].Though such approaches have shown nice promise, none of them has made a construct that meets all the standards printed higher than to expose tissues to such mechanical stimulation, a good array of bioreactors systems has been developed that permit investigators to review tissue growth and behavior underneath physiological conditions. Bioreactors aren't restricted to providing mechanical stimulation however are often accustomed enhance nutrient and $\mathrm{O}$ delivery similarly as facilitate semi-permanent culture and scale-up. Such systems are often easy devices accustomed mimic one facet of the vas setting, like cyclic distension of the tube-shaped structure wall, or a lot of elaborate systems that get to mimic the tube-shaped structure setting. All such systems have incontestable their ability to boost tissue growth by enhancing EW synthesis and/or increasing cell proliferation, similarly as a minimum of part restoring cells to their traditional in vivo phenotypes.

\section{Flow Conditioning of ECs}

The vary of vessel responses to flow-induced shear stresses is very broad and sometimes complicated. Though shear stress is directly perceived solely by the epithelial tissue, these signals are transmitted to different regions of the vessel wall and therefore the blood by the ECs via variety of physical and chemical pathways. During this manner, the whole vessel is capable of responding to changes in shear stress. These signals will induce changes in vessel diameter and tone, SMC proliferation, lumen thrombogenicity, and electronic countermeasures organization, all of that are essential to maintaining tube equilibrium. Specifically, traditional levels of shear stress decrease vessel thrombogenicity, maintain correct tube tone, and inhibit SMC proliferation, whereas low levels of shear stress promote membrane thickening and increase coagulum formation on the vessel lumen. Though the mechano transduction cascade isn't well understood, it's clear that the body structure, notably simple protein filaments, plays the principal role within the transmission of mechanical signals. ECs are shown to align within the flow direction with marked changes in cytoskeletal87 and sub-endothelial matrix organization. These changes in cytoskeletal organization and morphology are probably to own a significant impact on the power of ECs to sense and transmit shear 
Page 3 of 3

stress signals. Flow is incapable of inducement changes in European Community orientation and, in most cases, has no impact on European Community perform. In distinction, bedded flows are capable of inducement changes in European Community orientation, morphology, and performance over a spread of shear stresses. Finally, the power to manage transport of fluid and macromolecules across the vessel wall is essentially controlled by the epithelial tissue via a extremely regulated method that involves each convective and disseminative pathways $[16,17]$ which might be modulated by many organic chemistry and physical factors, notably shear stress. In vitro studies have shown that one dyne/cm2 of shear stress causes a 4 -fold increase in tube porosity, and ten dynes $/ \mathrm{cm} 2$ increase porosity 10 -fold, each of that are time dependent and reversible.

\section{Conclusion}

Although sure mixtures of cells (terminally differentiated or stemcell derived), scaffolds (synthetic or natural), stimulation (chemical, mechanical, and presumably electromagnetic), and tissue culture/ bioreactor systems (diffusive or convective transport) that are however to be known can little doubt result in enhancements, a significant obstacle to the advancement of the sector is that the current use of invasive or damaging strategies to watch most tissue growth variables (ie, physiological condition, localized ECM composition and microstructure, localized concentrations of soluble and certain factors, material and mechanical properties). The event of imaging strategies to create these measurements noninvasively would greatly improve the productivity of such studies by revealing spatiotemporal growth patterns and relationships among these variables that are elusive with current strategies. High-throughput models that accurately mirror tissue growth in constructs of the target size and pure mathematics would additionally enhance productivity. Ultimately, a prophetical basis for the best combination of cell source/scaffold/stimulation/ bioreactor can devolve on a additional complete understanding of however the cell integrates the assorted signals at the cellular and molecular level. This understanding can translate into biophysical models that relate cell cycle regulation and therefore the production and assembly of ECM parts in response to those integrated signals and ultimately into multiscale mechanical models that relate the evolving ECM at the molecular model to macroscopic mechanical and purposeful properties. There are recent times mechanical models of tube-shaped structure growth and transforming that are aimed during this direction.

\section{References}

1. American Heart Association Heart Disease and Stroke Statistics - 2005 Update. March 11, 2005.
2. Conte MS (1998) The ideal small arterial substitute: a search for the Holy Grail? FASEB J. 12: 43-45.

3. Nerem RM, Ensley AE (2004) The tissue engineering of blood vessels and the heart. Am J Transplant 4 (suppl 6): 36-42.

4. Teebken OE, Haverich A (2002) Tissue engineering of small diameter vascular grafts. Eur J Vasc Endovasc Surg. 23: 475-485.

5. Mayer JE Jr, Shin'oka T, Shum-Tim D (1997) Tissue engineering of cardiovascular structures. Curr Opin Cardiol. 12: 528-532.

6. Tranquillo RT (2002)The tissue-engineered small-diameter artery. Ann N Y Acad Sci. 961: 251-254.

7. Niklason LE (1999)Techview: medical technology. Replacement arteries made to order. Science286: 1493-1494.

8. Schmidt CE, Baier JM (2000)Acellular vascular tissues: natural biomaterials for tissue repair and tissue engineering. Biomaterials21: 2215-2231.

9. Sung HW, Hsu CS, Chen HC, Hsu HL, Chang Y, et al (1997) Fixation of various porcine arteries with an epoxy compound. Artif Organs. 21: 5058.

10. Robotin-Johnson MC, Swanson PE, Johnson DC, Schuessler RB, Cox JL (1998) An experimental model of small intestinal submucosa as a growing vascular graft. J ThoracCardiovascSurg116: 805-811.

11. Voytik-Harbin SL, Brightman AO, Kraine MR, Waisner B, Badylak SF (1997) Identification of extractable growth factors from small intestinal submucosa. J Cell Biochem. 67: 478-491.

12. Kim BS, Nikolovski J, Bonadio J, Smiley E, Mooney DJ (1999) Engineered smooth muscle tissues: regulating cell phenotype with the scaffold. Exp Cell Res. 251: 318-328.

13. Shum-Tim D, Stock U, Hrkach J, Shinoka T, Lien J, et al. (1999) Tissue engineering of autologous aorta using a new biodegradable polymer. Ann Thorac Surg. 68: 2298-2305.

14. Watanabe M, Shin'oka T, Tohyama S, Hibino N, Konuma T (2001) Tissue-engineered vascular autograft: inferior vena cava replacement in a dog model. Tissue Eng. 7: 429-439.

15. Canham PB, Talman EA, Finlay HM, Dixon JG (1991) Medial collagen organization in human arteries of the heart and brain by polarized light microscopy. Connect Tissue Res. 26: 121-134.

16. Weinberg CB, Bell E (1986)A blood vessel model constructed form collagen and cultured vascular cells. Science 231: 397-400.

17. Hirai J, Matsuda T (1995)Self-organized, tubular hybrid vascular tissue composed of vascular cells and collagen for low-pressure-loaded venous system. Cell Transplant. 4: 597-608.

18. Grassl ED, Oegema TR, Tranquillo RT (2002) Fibrin as an alternative biopolymer to type-I collagen for the fabrication of a media equivalent. J Biomed Mater Res. 60: 607-612. 